\title{
Quarterly repeat cycles of onabotulinumtoxinA in chronic migraine patients: the benefits of the prolonged treatment on the continuous responders and quality-of-life conversion rate in a real-life setting
}

\author{
Antonio Santoro $^{1} \cdot$ Andrea Fontana $^{2} \cdot$ Anna M. Miscio $^{1} \cdot{\text { Michele M. } \text { Zarrelli }^{1} \text { }}^{\text {. }}$ \\ Massimiliano Copetti ${ }^{2}$ - Maurizio A. Leone ${ }^{1}$
}

Received: 4 April 2017 / Accepted: 30 June 2017 /Published online: 19 July 2017

(C) The Author(s) 2017. This article is an open access publication

\begin{abstract}
OnabotulinumtoxinA was approved for treatment of chronic migraine (CM) after publication of Phase 3 Research Evaluating Migraine Prophylaxis Therapy (PREEMPT) trials. However, the PREEMPT trials lasted only up to 1 year. The main aim of our retrospective study was to evaluate whether a prolonged treatment of onabotulinumtoxinA (18 months, six quarterly cycles) will sustain or further improve the efficacy results and the quality of life achieved at 6 and 12 months. Patients were adults with $\mathrm{CM}$ with or without overuse of drugs, with at least six regularly repeat onabotulinumtoxinA treatments, administered according to the PREEMPT protocol. The outcomes were investigated after 6,12 , and 18 months of treatment with respect to baseline and with respect to each previous study time point. Headache days and hours, and dosage of headache medication taken with latency period, were collected from the patients daily. Quality of life was evaluated by means of the Migraine Disability Assessment (MIDAS) questionnaire. At each study time point, the proportion of responder patients with respect to baseline was evaluated. For all measures, the baseline
\end{abstract}

All the authors declare that they take full responsibility for the integrity of the data, the analyses, and the interpretation of the results. Results of this work have not been published elsewhere.

Antonio Santoro

antonio.santoro@operapadrepio.it

1 Unit of Neurology, IRCCS Casa Sollievo della Sofferenza, San Giovanni Rotondo, FG, Italy

2 Unit of Biostatistics, IRCCS Casa Sollievo della Sofferenza, San Giovanni Rotondo, FG, Italy data were referred to the previous month before starting. Forty-seven patients were evaluated. Our data show a decrease in the monthly headache days and hours, at each study evaluation, with respect to the previous one. They showed that beyond the first year, a statistically significant difference in the monthly days of headache compared at 18 vs. 12 months is observed. A significantly higher proportion of patients (with a response greater than $75 \%$ decrease from baseline in the frequency of headache days and hours) was observed at month 18 compared to month 12 . The proportion of patients in MIDAS grade I increased over time, and a statistically significant improvement in MIDAS I score was obtained from month 12 to month 18 . A positive modification in the consumption of analgesics over time was observed ( $p$ for trend <0.001). The mean acute drug latency strongly decreased over time. Our study confirmed that onabotulinumtoxinA is an effective treatment to reduce headache-related disability and improve patients' quality of life, highlighting that upon repeated administration, the therapy efficacy increases significantly and a progressive trend of "first-time response" is observed for the entire period under consideration.

Keywords Chronic migraine - Medication overuse headache · Migraine abuse $\cdot$ Preventative therapy $\cdot$ OnabotulinumtoxinA

\section{Background}

Chronic migraine (CM) is a common and debilitating neurological disorder affecting up to $2.4 \%$ of the general population and with an incidence estimated to be $2.5 \%$ per year [1]. 
According to the International Classification of Headache Disorders (ICHD-III), CM is defined as headache on 15 or more days per month for more than 3 months (at least 8 days should meet criteria for migraine without aura or respond to migraine-specific treatment) $[1,2]$.

$\mathrm{CM}$ is associated with significant disability and reduced health-related quality of life [3, 4]. Moreover, persons with $\mathrm{CM}$ have a greater economic burden than patients with episodic migraine (EM) [5-7].

Risk factors for the chronification of migraine have been identified, such as female gender, older age, Caucasian ethnicity, low socioeconomic status, comorbidity with other chronic diseases like obesity or psychiatric disorders, high-frequency EM, and overuse of symptomatic medication $[1,8]$.

Many patients with $\mathrm{CM}$ take a high amount of abortive medications. It is estimated that around $50-80 \%$ of patients with $\mathrm{CM}$ show an analgesic overuse that may induce medication overuse headache (MOH) [9]. There is no general agreement as to whether $\mathrm{MOH}$ is a consequence or a cause of CM [10]. As for CM, psychopathological comorbidities are often present among patients with $\mathrm{MOH}$, and quality of life is highly related to $\mathrm{CM}$ as well as to relapse into $\mathrm{MOH}[11,12]$.

Currently, onabotulinumtoxinA is the unique drug specifically indicated for prophylaxis of headache in adult patients with CM [13]. It is the only therapy with an approved indication [14]. Recently, the European Headache Federation recognized the value of onabotulinumtoxinA in CM prevention and specified that before labelling a patient as affected by refractory $\mathrm{CM}$, a proper treatment with this drug needs to be completed [15].

The onabotulinumtoxinA indication is based on the results of two large-scale, placebo-controlled, multicenter trials (PREEMPT (Phase 3 Research Evaluating Migraine Prophylaxis Therapy)).

The Pooled PREEMPT had a 28-day baseline screening phase and a 24-week, 2-cycle, double-blind placebocontrolled, parallel-group phase with two injection cycles, followed by a 32-week, open-label phase with three injection cycles and demonstrated the efficacy, safety, and tolerability of repeated treatment with 155-195 U of onabotulinumtoxinA, every 12 weeks over 56 weeks (up to five treatment cycles), as a prophylactic treatment for CM in adults [16-19].

Nowadays, few studies have been published based on onabotulinumtoxinA real-life efficacy and few data are available on its efficacy beyond the fifth cycle of treatment administered over a period longer than 12 months [20-23].

The main aim of our retrospective study was to evaluate whether in a real-life clinical setting, a prolonged treatment of onabotulinumtoxinA (18 months, six quarterly cycles) will sustain or further improve the efficacy results and the quality of life achieved at 6 and 12 months.

\section{Methods}

\section{Study cohort}

All patients included in our retrospective study were adults, male and female, with $\mathrm{CM}$ defined by the International Classification of Headache Disorders (ICHD-3 beta 2013) [24], with (the majority) or without overuse of drugs, with at least six regularly repeat onabotulinumtoxinA treatments.

Our patient cohort had received and failed other preventive therapies due to lack of efficacy or intolerable side effects and was able to fill a specific "migraine diary," without lack of information.

Exclusion criteria were pregnancy or breastfeeding, symptoms of psychiatric disease, and history of botulinum toxin use for other clinical purposes.

To evaluate a cohort reflecting a real-life clinical setting as closely as possible, patients receiving any preventive or symptomatic therapy of migraine were not excluded from the analysis.

The data were collected from IRCCS Casa Sollievo della Sofferenza-San Giovanni Rotondo-S.C. di Neurologia (from April 2013 to August 2016).

The work carried out complies with the Declaration of Helsinki and was approved by Sezione del CE IRCCS Istituto Tumori Giovanni Paolo II di Bari presso la Fondazione Casa Sollievo della Sofferenza di San Giovanni Rotondo ICF: V1.0_07 APR 2015.

Each patient signed a free informed consent for the analysis and publication of the data.

\section{Treatment}

OnabotulinumtoxinA was injected in a day hospital setting, every 3 months ( \pm 10 days) as per the PREEMPT protocol (i.e., 155-195 U onabotulinumtoxinA in 31-39 sites).

\section{Clinical assessment}

All patients were trained to complete a specific headache diary, and they were asked to fill it out at baseline and continuously after receiving onabotulinumtoxinA. Headache days, cumulative hours of headache in headache days, and dosage of headache medication intake, with latency time (time from symptomatic drug administration to the analgesic effect) were daily collected from the patients in the diary and were evaluated by the investigator at each quarterly visit.

The quality of life was evaluated by means of the Migraine Disability Assessment (MIDAS) questionnaire [25] administered at baseline and at each quarterly visit (3 months after each treatment session).

At each visit, the proportion of responder patients with respect to baseline was evaluated. 
For all measures, the baseline data were referred to the previous month before starting onabotulinumtoxinA.

\section{Outcome assessment}

The following outcomes were investigated after 6 (T6-i.e., after II cycles), 12 (T12 - i.e., after IV cycles) and 18 months (T18 - i.e., after VI cycles) of treatment with respect to baseline visit (T0) and with respect to each previous time point (T12 vs. T6; T18 vs. T12):

1. Changes in monthly days and hours of headache

2. Consumption and latency time (in hours) of analgesics

3. MIDAS grade distribution

Moreover, patient treatment responsiveness in terms of the relative reduction (i.e., percentage of responsiveness) for the number of days and hours of headache at T6, T12, and T18 with respect to baseline (T0) and with respect to each study time was investigated.

Such percentage was calculated by subtracting the value of the outcome at $\mathrm{T} 0$ with the value of the outcome at follow-up and dividing such difference by the value of the outcome at T0. According to such percentage, patients were therefore classified as follows:

- Non-responder $<30 \%$ reduction

- Partial responder $\geq 30$ and $<50 \%$ reduction

- $\quad$ Responder $\geq 50$ and $\leq 75 \%$ reduction

- High responder $>75 \%$ reduction

\section{Statistical analysis}

Patient characteristics were reported as mean \pm standard deviation (SD), median along with lower-upper quartiles (q1-q3) and range (min-max), or frequencies and percentages, for continuous and categorical variables, respectively.

Changes in monthly number of days and hours of headache, MIDAS distribution consumption of analgesics, and drug latency over follow-up time (i.e., at T0, T6, T12, and T18) were assessed using the hierarchical generalized linear model (HGLM) for longitudinal data, for each outcome at issue. Within this framework, the Poisson distribution was assumed to model continuous outcomes concerned with counts (i.e., monthly number of days and hours of headache, drug latency in hours) whereas the logistic distribution was assumed to model binary outcomes (i.e., consumption of analgesics). Changes over time in the distribution of multinomial variables (i.e., MIDAS and responders' groups) were assessed performing HGLMs with logistic distribution, using indicator variables (i.e., dummy variables) for each category of the variable at issue. The first-order autoregressive covariance structure was used to account for the correlation between repeated measurements over time. Estimated means (or percentages for categorical variables) were carried out from HGLMs and were reported along with their $95 \%$ confidence interval (95\% CI), including follow-up time as categorical covariate. For each HGLM, a test for overall difference over time was assessed by looking at the significance of the type III test, whereas pairwise comparisons were assessed as statistical contrasts and were adjusted following Benjamini-Hochberg step-down procedure. The presence of a linear trend for the estimated means (or proportions) over time was assessed by looking at the significance of the regression coefficient of the follow-up time variable, included into the model as continuous covariate into HGLMs ( $p$ for trend). For each continuous outcome at issue, longitudinal plots of the estimated means over time were further reported whereas for each categorical outcome at issue, histograms of the estimated percentages were reported instead. All plots were represented along with error bars which represented $95 \%$ CI.

Two-sided $p$ values $<0.05$ were considered for statistical significance. All analyses were performed using SAS Software, Release 9.4 (SAS Institute, Cary, NC, USA) and R (package: ggplot2).

\section{Results}

From April 2013 to August 2016, a total of 207 patients with $\mathrm{CM}$ received at least one administration cycle of onabotulinumtoxinA according to the PREEMPT protocol (treatments quarterly, 155-195 U onabotulinumtoxinA in 31-39 sites). Twenty-eight patients discontinued the treatment due to improvement ( $\leq 4$ headache days and responder to symptomatic treatment for 3 months) before the sixth cycle, and 67 patients were lost to follow-up. Sixty-five patients were ongoing (i.e., before VI onabotulinumtoxinA cycle) when the database was locked for this analysis (August 31, 2016). Finally, 47 patients received six treatments quarterly during 18 months. So, our cohort consisted of 47 patients (37 females, $78.7 \%$ ) with a mean age of $48.2 \pm 13.6$ years (range 41-59). Patients had a diagnosis of CM for a mean of $9.4 \pm 6.8$ years (range $1-30$ ).

Demographic and treatment baseline patient characteristics are reported in Table 1.

\section{Change of headache days and hours/month}

Results from comparisons of monthly mean of headache days at each time point, as well as from comparisons of monthly mean of headache hours, are reported in Table 2. 
Table 1 Demographic details of study population at baseline (47 patients)

\begin{tabular}{lll}
\hline Age (years) & Mean \pm SD & $48.2 \pm 13.6$ \\
& Median $(\mathrm{q} 1-\mathrm{q} 3)$ & $47(41-59)$ \\
& Range & $18-72$ \\
Sex-females & $n(\%)$ & $37(78.7)$ \\
Years of chronic headache & Mean \pm SD & $9.4 \pm 6.8$ \\
& Median (q1-q3) & $9(4-12)$ \\
Patients assuming NSAIDs & Range & $1-30$ \\
Patients assuming triptans & $n(\%)$ & $27(57.5)$ \\
Patients assuming other drugs & $n(\%)$ & $23(48.9)$ \\
& $n(\%)$ & $18(38.3)$ \\
\hline
\end{tabular}

The mean of monthly headache days significantly decreased from $25.9 \pm 5.3$ at baseline to $11.5 \pm 8.8$ after II onabotulinumtoxinA cycle (T6), to $9.6 \pm 6.8$ after IV onabotulinumtoxinA cycle (T12), down to reaching $6.3 \pm 5.7$ after VI onabotulinumtoxinA cycle (T18) ( $p$ for trend $<0.001$ ). Moreover, a statistically significant decrease of monthly headache days was observed after 6 months with respect to baseline $(p<0.001)$ and after 18 months with respect to 12 months $(p=0.001)$. Also, the mean of monthly headache hours significantly decreased over time (T0, $547.7 \pm 183.3 ; \mathrm{T} 6,173.4 \pm 195.3 ; \mathrm{T} 12,90.4 \pm 93.9$; $\mathrm{T} 18,53.2 \pm 79.2$ ) ( $p$ for trend <0.001). In this case, statistically significant pairwise comparisons were found contrasting all time points: T6 vs. T0 $(p<0.001)$, T12 vs. T6 $(p<0.001)$, and T18 vs. T12 ( $p=0.013)$.

Plots of monthly headache days and hours means over follow-up time are reported in Fig. 1a, b, respectively.

\section{Consumption of analgesics and change in mean latency time after acute drug intake}

The study results showed also a positive modification in the consumption of analgesics over time (Table 3 ), pointing out a great decrease of painkiller dosages for each drug class ( $p$ for trend $<0.001$ ).

This was also corroborated with an important and significant decrease in monthly mean time before the effects of any symptomatic medication. The mean acute drug latency strongly decreased at T6 vs. T0 $(p<0.001)$ and at T12 vs. T6 $(p=0.001)$, showing a further slight improvement at T18 (Table 4 and Fig. 2).

\section{Distribution of patient response to treatment}

Distribution of responder groups (in terms of improvement in monthly days and cumulative hours of headache) at each time point is summarized in Table 5 and is graphically represented in Fig. 3. We observed a strong increase in the proportion of responder patients already after 6 months on either days or hours of headache. As for the monthly days, when classifying responder patients into the three defined categories, we found a significant increase in the proportion of high responders from T12 (34.1\%) to $\mathrm{T} 18$ (57.4\%) $(p=0.009)$. A different scenario was observed when referring to the monthly hours of headache: the proportion of high responders for the hours of headache significantly increased already at T12 vs. T6 (from $53.2 \%$ at $\mathrm{T} 6$ to $76.6 \%$ at $\mathrm{T} 12 ; p=0.009$ ), and a further enhancement was observed at 18 months $(89.4 \%)$.

Table 2 Monthly headache days and hours at baseline (T0) and after 6 (T6), 12 (T12), and 18 months (T18)

\begin{tabular}{|c|c|c|c|c|c|}
\hline & & $\mathrm{T} 0$ & $\mathrm{~T} 6$ & $\mathrm{~T} 12$ & $\mathrm{~T} 18$ \\
\hline \multirow[t]{3}{*}{ Monthly days with headache } & Mean \pm SD & $25.9 \pm 5.3$ & $11.5 \pm 8.8$ & $9.6 \pm 6.8$ & $6.3 \pm 5.7$ \\
\hline & Median (q1-q3) & $30(20-30)$ & $9(4-18)$ & $8(5-14)$ & $5(2-8)$ \\
\hline & $\begin{array}{l}\text { Range } \\
95 \% \mathrm{CI} \\
\mathrm{n}\end{array}$ & $\begin{array}{l}15-30 \\
23.0-29.1 \\
47\end{array}$ & $\begin{array}{l}0-30 \\
9.6-13.8 \\
47\end{array}$ & $\begin{array}{l}0-30 \\
8.0-11.7 \\
47\end{array}$ & $\begin{array}{l}0-30 \\
5.0-8.0 \\
47\end{array}$ \\
\hline \multirow[t]{3}{*}{ Monthly hours with headache } & Mean \pm SD & $547.7 \pm 183.4$ & $173.4 \pm 195.3$ & $90.4 \pm 93.9$ & $53.2 \pm 79.2$ \\
\hline & Median (q1-q3) & $600(400-700)$ & $100(30-250)$ & $60(20-120)$ & $25(12-50)$ \\
\hline & $\begin{array}{l}\text { Range } \\
95 \% \mathrm{CI} \\
\mathrm{n}\end{array}$ & $\begin{array}{l}112-720 \\
478.4-627.0 \\
47\end{array}$ & $\begin{array}{l}0-700 \\
136.3-220.5 \\
47\end{array}$ & $\begin{array}{l}0-400 \\
64.8-126.1 \\
47\end{array}$ & $\begin{array}{l}0-350 \\
34.5-82.2 \\
47\end{array}$ \\
\hline \multicolumn{6}{|c|}{$\begin{array}{l}p \text { values from HGLM and pairwise } \\
\text { comparisons }\end{array}$} \\
\hline Outcome & $p$ for overall difference & T6 vs. T0 & T12 vs. T6 & T18 vs. T12 & $p$ for trend \\
\hline Monthly days with headache & $<0.001$ & $<0.001$ & 0.072 & 0.001 & $<0.001$ \\
\hline Monthly hours with headache & $<0.001$ & $<0.001$ & $<0.001$ & 0.013 & $<0.001$ \\
\hline
\end{tabular}



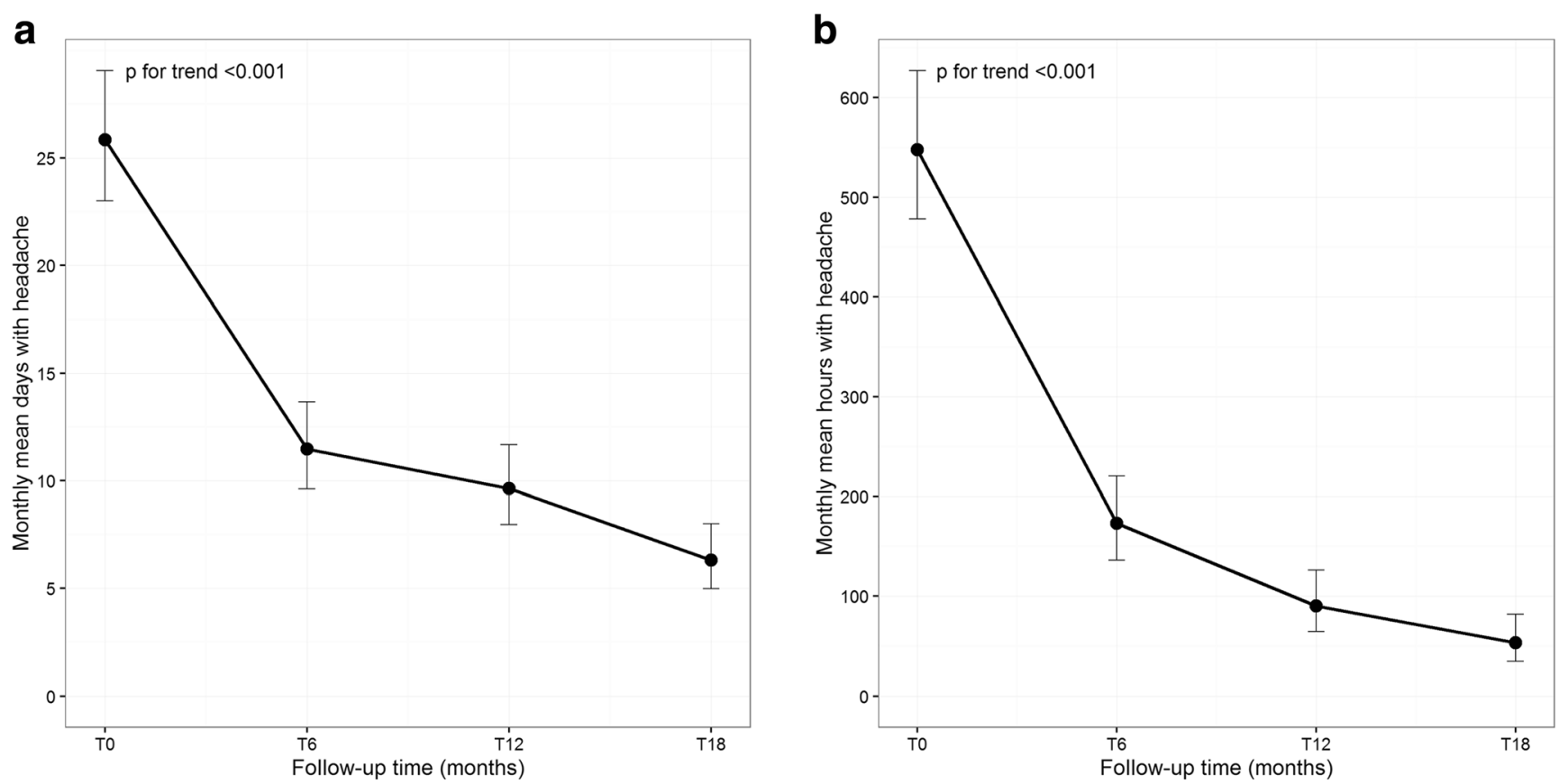

Fig. 1 Plots of monthly headache days (a) and hours (b) means at baseline (T0) and after 6, 12, and 18 months (T6, T12, and T18) of treatment for onabotulinumtoxinA (six quarterly cycles). Error bars represent the $95 \%$ confidence interval around the means

\section{MIDAS grade distribution}

MIDAS grade distribution is represented at each time point in Table 6 and plotted in Fig. 4. The results suggest a significant progressive improvement from baseline to 6-12-18 months, pointing out a high and significant decrease of patients with grade IV already at 6 months compared with baseline $(p<0.001)$, and a constant clinical improvement was confirmed in the subsequent visits ( $p$ for trend <0.001). At 18 months, a statistically significantly higher proportion of patients with MIDAS grade I compared to those at 12 months (T12, 34.0\%; T18, 55.3\%; $p=0.022$ ) was observed. Conversely, a statistically significantly lower proportion of patients with MIDAS grade III resulted (T12, 34.0\%; $\mathrm{T} 18,10.6 \% ; p=0.017)$.

Table 3 Distribution of symptomatic drug (i.e., analgesics) dosage units among users

\begin{tabular}{|c|c|c|c|c|c|}
\hline & & T0 & T6 & $\mathrm{T} 12$ & $\mathrm{~T} 18$ \\
\hline NSAID use (total number of dosage units) & $\begin{array}{l}\text { Mean } \pm \text { SD } \\
\text { Median (q1-q3) } \\
\text { Range } \\
95 \% \text { CI } \\
\text { Users }\end{array}$ & $\begin{array}{l}39.7 \pm 31.3 \\
30(20-55) \\
3-120 \\
30.6-49.5 \\
27\end{array}$ & $\begin{array}{l}11.8 \pm 15.9 \\
4(3-15) \\
2-70 \\
7.8-18.7 \\
25\end{array}$ & $\begin{array}{l}8.2 \pm 9.0 \\
4.5(2.5-11.5) \\
1-40 \\
4.7-13.2 \\
28\end{array}$ & $\begin{array}{l}5.4 \pm 6.6 \\
3(2-6) \\
1-30 \\
2.8-10.0 \\
28\end{array}$ \\
\hline Triptan use (total number of dosage units) & $\begin{array}{l}\text { Mean } \pm \text { SD } \\
\text { Median (q1-q3) } \\
\text { Range } \\
95 \% \text { CI } \\
\text { Users }\end{array}$ & $\begin{array}{l}30.4 \pm 26.4 \\
24(15-30) \\
7-120 \\
21.3-41.7 \\
23\end{array}$ & $\begin{array}{l}21.9+30.6 \\
9(5-22) \\
2-120 \\
12.0-29.2 \\
17\end{array}$ & $\begin{array}{l}8.6 \pm 9.5 \\
5.5(2.5-10) \\
1-35 \\
4.4-16.1 \\
20\end{array}$ & $\begin{array}{l}7.6 \pm 8.1 \\
5(3-9) \\
1-30 \\
3.0-15.0 \\
15\end{array}$ \\
\hline Other drugs use (total number of dosage units) & $\begin{array}{l}\text { Mean } \pm \text { SD } \\
\text { Median (q1-q3) } \\
\text { Range } \\
95 \% \text { CI } \\
\text { Users }\end{array}$ & $\begin{array}{l}40.1 \pm 37.0 \\
30(9-50) \\
3-120 \\
28.2-54.0 \\
18\end{array}$ & $\begin{array}{l}11.9 \pm 16.2 \\
6(3-12) \\
1-70 \\
7.2-21.4 \\
19\end{array}$ & $\begin{array}{l}6.6 \pm 6.7 \\
4.5(2.10) \\
1-30 \\
4.2-15.5 \\
22\end{array}$ & $\begin{array}{l}5.1 \pm 5.8 \\
3(2-6) \\
1-22 \\
1.7-13.3 \\
13\end{array}$ \\
\hline \multicolumn{6}{|l|}{$p$ values from HGLM and pairwise comparisons } \\
\hline Outcome & $p$ for overall difference & T6 vs. T0 & T12 vs. T6 & T18 vs. T12 & $p$ for trend \\
\hline NSAID use (total number of dosage units) & $<0.001$ & $<0.001$ & 0.195 & 0.205 & $<0.001$ \\
\hline Triptan use (total number of dosage units) & $<0.001$ & 0.015 & 0.005 & 0.483 & $<0.001$ \\
\hline Other drugs use (total number of dosage units) & $<0.001$ & $<0.001$ & 0.241 & 0.241 & $<0.001$ \\
\hline
\end{tabular}


Table 4 Latency after intake of any symptomatic therapy, among patients who were treated with analgesics (i.e., users)

\begin{tabular}{llllll}
\hline & & T0 & T6 & T12 & T18 \\
Latency time (h) & Mean \pm SD & $5.9 \pm 0.4$ & $2.9 \pm 1.5$ & $2.1 \pm 1.4$ & $2.0 \pm 1.5$ \\
& Median (q1-q3) & $6(6-6)$ & $3(2-4)$ & $2(1-3)$ & $1.5(1-2)$ \\
& Range & $4-6$ & $1-6$ & $1-6$ & $1-6$ \\
& $95 \%$ CI & $5.4-6.5$ & $2.5-3.4$ & $1.8-2.5$ & $1.7-2.4$ \\
& Users & 47 & 46 & 47 & 44 \\
$p$ values from HGLM and pairwise comparisons & & & & \\
\multirow{2}{*}{ Latency time (h) } & $\begin{array}{l}\text { p for overall difference } \\
\text { < }\end{array}$ & T6 vs. T0 & T12 vs. T6 & T18 vs. T12 & $p$ for trend \\
& $<0.001$ & $<0.001$ & 0.001 & 0.654 & $<0.001$ \\
\hline
\end{tabular}

\section{Safety}

During the onabotulinumtoxinA exposure, the unique reported treatment-related adverse event (AE) was neck pain. It occurred in only two patients from T0 to T12.

At baseline, six patients had received the endovenous treatment as a consequence of headache. During the study, the same patients were occasionally hospitalized in the emergency room due to poor treatment efficacy, but only one after T12.

\section{Discussion}

Migraine is an episodic disorder, but in its natural course, its frequency, in a particular subset of patients, could progressively increase and evolve to a chronic form. The higher frequency of attacks can lead to a chronic intake of abortive medications as triptans and non-steroidal anti-inflammatory drugs (NSAIDs), both associated with a greater risk of developing cardiocerebrovascular events and renal events [26, 27]. Thus, $\mathrm{CM}$ is a serious and debilitating neurological disorder with high risk of medication overuse given by the frequent partial response to treatment, both abortive and preventive. Therefore, the opportunity to provide new effective therapeutic options to patients represents a crucial step in CM treatment [28]. The PREEMPT clinical trials, the largest (1384 patients) studies in CM, showed that onabotulinumtoxinA is a safe, well-tolerated, and effective prophylactic therapy for CM patients [18-20].
Fig. 2 Trend of latency time (h) means after symptomatic drug administration at baseline (T0) and after 6,12 , and 18 months (T6, T12, and T18) of treatment for onabotulinumtoxinA. Error bars represent $95 \%$ confidence intervals around the means

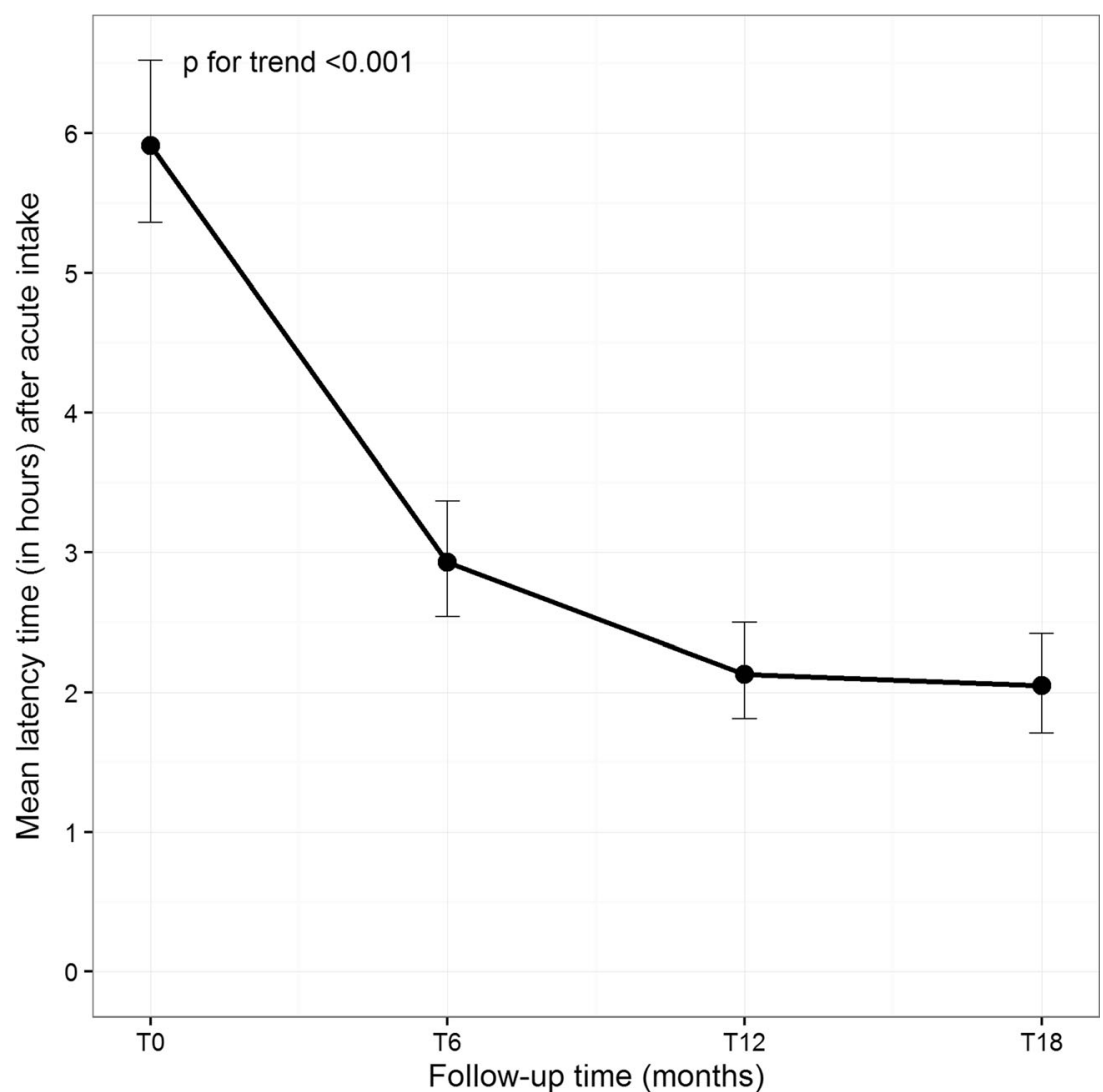




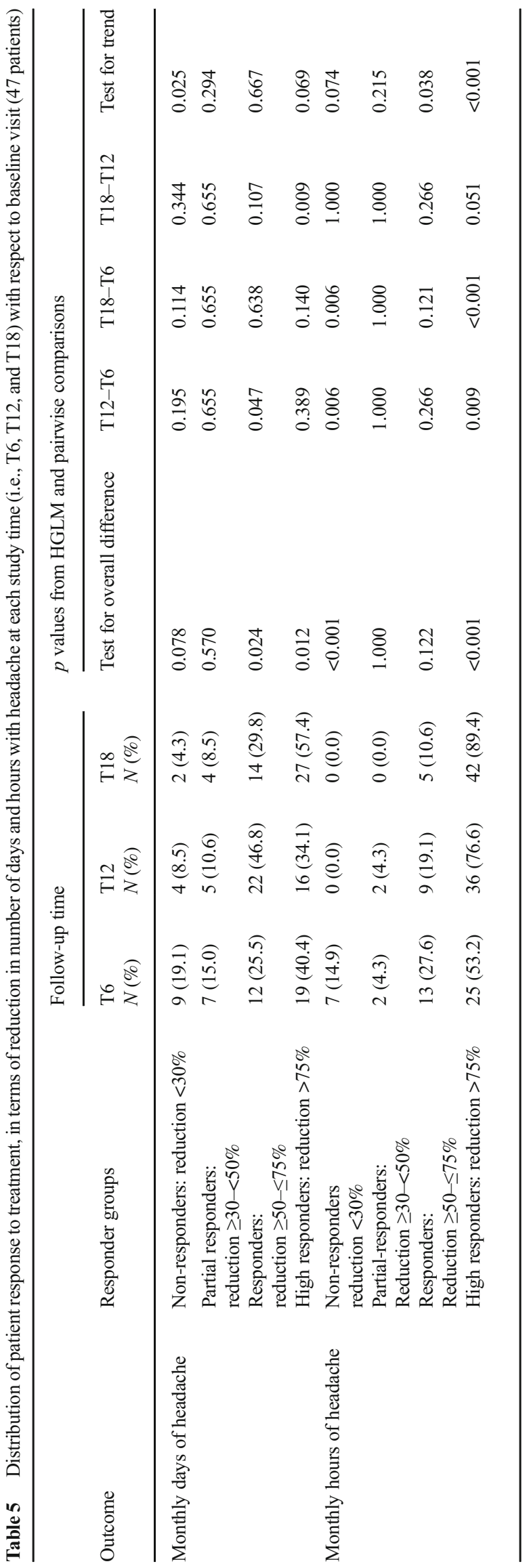

The notable results led to the worldwide specific indication of onabotulinumtoxinA for the prevention of headache in $\mathrm{CM}$ patients $[17-19,29,30]$.

Few studies in a real-life setting have been recently published and confirm the efficacy and safety of onabotulinumtoxinA [31-34]. Importantly among these, Negro et al. $[18,19]$ have proved in routinary practice that onabotulinumtoxinA can be safely used for long-term treatment of MOH comorbidity in CM. Furthermore, Guerzoni et al. [22] have described that therapy discontinuation leads to a general worsening of health-related quality of life.

Our retrospective study provides data from patients treated with onabotulinumtoxinA, administered over 18 months with six quarterly cycles, in a real-life setting. In our analysis, the efficacy of onabotulinumtoxinA is evaluated time by time, in addition to the comparison with baseline.

Our data show a significant decrease in the monthly headache days and hours, at each study evaluation, with respect to previous ones. They showed that beyond the first year, a statistically significant difference in the monthly days of headache comparing T18 vs. T12 is observed, so confirming that repeated injections over time might give much better results in support of observations by Negro et al. [20, 21].

There are still questions open in the onabotulinumtoxinA treatment for $\mathrm{CM}$, such as the possible superior efficacy of symptomatic medication effects after repeated cycles.

Nevertheless, an increased sensitization in pain processing [35] has been described in patients with medication overuse; through the inhibition of peripheral sensitization [36], also onabotulinumtoxinA may influence central mechanisms responsible for facilitation in pain processing [37]. For the first time, our study evaluated the changes of the overall drug intake (in units of drug consumption) and the latency time of symptomatics during the onabotulinumtoxinA cycles, suggesting that repeated cycles of onabotulinumtoxinA decrease significantly the dosage of any of them and very importantly also the latency time period ( $p$ for trend $<0.001$ ). This represents an important preliminary finding since reduction in the use of pain medication and abuse might also constitute an essential factor reducing cardiovascular risk in patients affected with CM [26].

Further analyses are ongoing in order to evaluate the drug reduction by amount and therapeutic class and/or their association to assess potential similarities and/or differences in benefits.

The PREEMPT study efficacy analyses included the proportion of patients with a $50 \%$ or more decrease from baseline in the frequency of headache days and, separately, headache episodes $[17,19]$. In our observation, we considered the mean reduction of monthly headache days and cumulative headache hours. Furthermore, similarly to our results, in the study by Silberstein et al. 2015 [30], a PREEMPT post hoc analysis showed that a meaningful proportion of patients with $\mathrm{CM}$ 


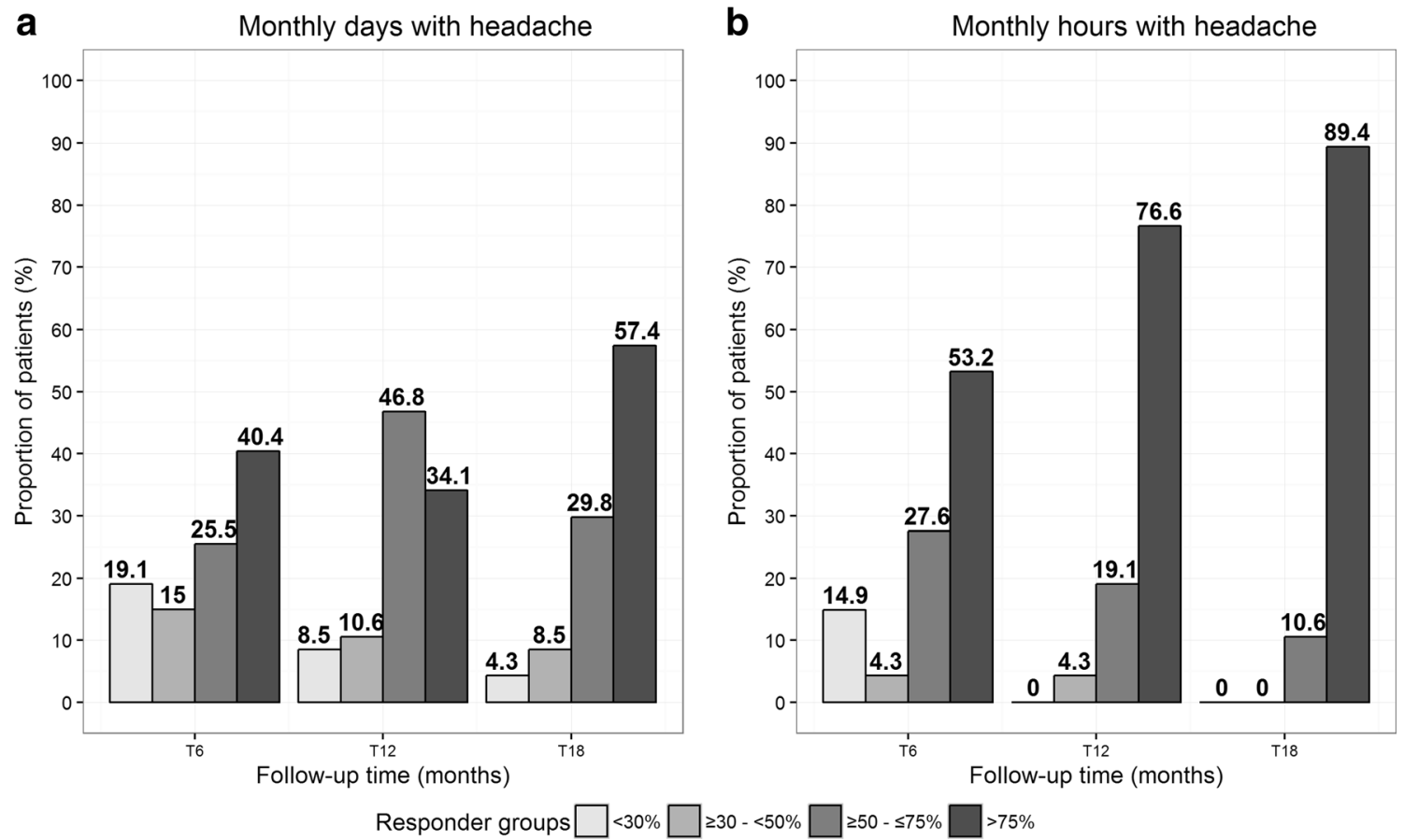

Fig. 3 Frequency distribution of patient response to treatment, in terms of number of monthly headache days (a) and hours (b) with headache at T6, T12, and T18 with respect to baseline visit

treated with onabotulinumtoxinA who did not respond to the first treatment cycle responded in the second and third cycles of treatment. Our findings confirmed a progressive and remarkable trend of "first-time response" upon repeat treatments. In fact, we observed a high conversion rate from patients defined as "non-responders" and "partial responders" to "responders" and "high responders" throughout the period under consideration.

We assessed how many patients showed a reduction of the symptoms between 50 and $75 \%$ and how many patients were high responders (reduction $>75 \%$ ), at each evaluation time. In our analysis, a significantly higher percentage of patients had a more than $75 \%$ decrease from baseline in the frequency of headache days and hours, improving over the time, which supports the evidence that the benefits of regularly repeated treatment are meaningful to the patients (proportion of high-responder patients at T18 in headache days: $57.4 \%$; T18 vs. T12 $p=0.009$; proportion of high-responder patients at $\mathrm{T} 18$ in headache hours: $89.4 \%$; T18 vs. T12 $p=0.051$ ).
Table 6 Patient distribution as per MIDAS grades at each study time (47 patients)

\begin{tabular}{|c|c|c|c|c|c|c|}
\hline & & $\mathrm{T} 0$ & $\mathrm{~T} 6$ & & $\mathrm{~T} 12$ & $\mathrm{~T} 18$ \\
\hline \multirow[t]{2}{*}{ MIDAS grade I } & $n(\%)$ & $0(0.0)$ & $17(36.2)$ & & $16(34.0)$ & $26(55.3)$ \\
\hline & $95 \% \mathrm{CI}$ & $0-100$ & $25.2-48.9$ & & $23.3-46.7$ & $42.8-67.2$ \\
\hline \multirow[t]{2}{*}{ MIDAS grade II } & $n(\%)$ & $0(0.0)$ & $11(23.4)$ & & $12(25.5)$ & $15(31.9)$ \\
\hline & $95 \% \mathrm{CI}$ & $0-100$ & $14.4-35.6$ & & $16.2-37.9$ & $21.5-44.6$ \\
\hline \multirow[t]{2}{*}{ MIDAS grade III } & $n(\%)$ & $14(29.8)$ & $12(25.5)$ & & $16(34.0)$ & $5(10.6)$ \\
\hline & $95 \% \mathrm{CI}$ & $18.3-44.5$ & $15.0-40.1$ & & $21.8-48.8$ & $4.4-23.4$ \\
\hline \multirow[t]{2}{*}{ MIDAS grade IV } & $n(\%)$ & $33(70.2)$ & 7 (14.9) & & $3(6.4)$ & $1(2.1)$ \\
\hline & $95 \% \mathrm{CI}$ & $55.5-81.7$ & $7.2-28.4$ & & $2.0-18.3$ & $0.3-14.1$ \\
\hline \multicolumn{7}{|c|}{$p$ values from HGLM and pairwise comparisons } \\
\hline \multicolumn{2}{|l|}{ Outcome } & $\begin{array}{l}\text { Test for overall } \\
\text { difference }\end{array}$ & T6 vs. T0 & T12 vs. T6 & T18 vs. T12 & $p$ for trend \\
\hline \multicolumn{2}{|l|}{ MIDAS grade I } & 0.047 & 0.998 & 0.998 & 0.022 & $<0.001$ \\
\hline \multicolumn{2}{|l|}{ MIDAS grade II } & 0.740 & 0.998 & 0.998 & 0.998 & $<0.001$ \\
\hline \multicolumn{2}{|l|}{ MIDAS grade III } & 0.043 & 0.614 & 0.614 & 0.017 & 0.081 \\
\hline \multicolumn{2}{|l|}{ MIDAS grade IV } & $<0.001$ & $<0.001$ & 0.211 & 0.233 & 0.003 \\
\hline
\end{tabular}


Fig. 4 Frequency distribution for MIDAS grade at baseline (T0) and after 6,12 , and 18 months (i.e., T6, T12, and T18). Error bars represent $95 \%$ confidence interval around proportions

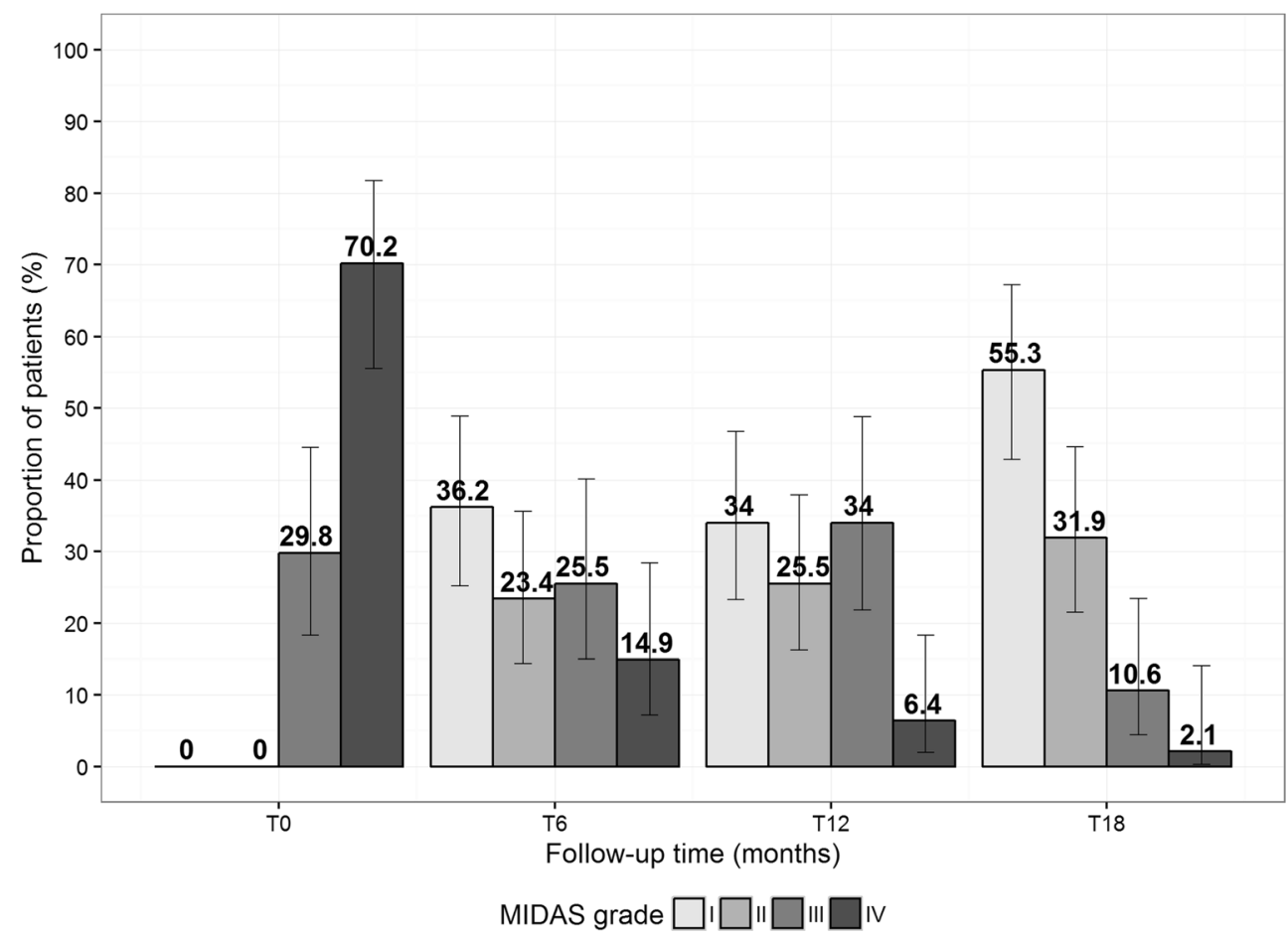

The benefits of the therapy found additional evidences also in terms of improvement in the quality of life.

Patients were asked to complete a MIDAS questionnaire. The MIDAS grade is based on responses to five questions about disability associated with headache in the previous 3 months [25]. The MIDAS is used in clinical research and in clinical practice. The reliability and validity have been demonstrated in a series of studies [38, 39]. Our quality-of-life evaluation includes the MIDAS GRADE patient distribution at each study time. The data confirm the efficacy results described above. The proportion of patients in MIDAS grade I (which corresponds to little or no disability) increases over time, with a statistically significant difference between 18 months and 12 months. At the same time, the proportion of patients in grade III (moderate disability) decreases significantly. Conversely, already at the T6, we observed a significant decrease of patients in grade IV (severe disability). Once again, the result suggests that there is a consistent trend of improvement with repeated injection.

The safety results that emerged from our study confirm the data of the previous studies [17-19].

\section{Conclusion}

Overall, our real-life results showed efficacy and safety of repeated cycles of onabotulinumtoxin A 155-195 U in patients affected with $\mathrm{CM}$ with or without overuse medication, thus confirming the efficacy data from previous randomized clinical trials for $\mathrm{CM}$ prophylaxis and pointing out that a prolonged treatment of onabotulinumtoxinA (18 months, six quarterly cycles) is able to sustain and further improve significantly the efficacy results and the quality of life achieved at 6 and 12 months.

$\mathrm{AE}$, adverse event; $\mathrm{CI}$, confidence interval; $\mathrm{CM}$, chronic migraine; HGLM, hierarchical generalized linear model; ICHD-III (beta), International Classification of Headache Disorders, Third Edition-BETA Version; MOH, medication overuse headache; NSAIDs, non-steroidal anti-inflammatory drugs; PREEMPT, Phase III Research Evaluating Migraine Prophylaxis Therapy; q1-q3, lower-upper quartiles; SD, standard deviation

Acknowledgements Native English check and Publication fees for this manuscript were supported by Allergan SpA-Italy.

Authors' contributions AS: idea of the study, patients' diagnoses and follow-ups, performed all the injections, data acquisition/interpretation and coordination of activities and manuscript preparation.

AMM: data collection and coordination of some activities.

$\mathrm{MC}$ and $\mathrm{AF}$ : statistical analysis, interpretation of data, and manuscript drafting/revision.

MAL and MMZ: idea of the study, interpretation of data, and manuscript drafting/revision.

All the authors have read and approved the final manuscript.

\section{Compliance with ethical standards}

Ethics approval and consent to participate The protocol was approved by Sezione del CE IRCCS Istituto Tumori Giovanni Paolo II di Bari presso la Fondazione Casa Sollievo della Sofferenza di San Giovanni Rotondo ICF: V1.0_07 APR 2015. 
Consent for publication Each patient signed a free informed consent for the analysis and publication of the data.

Availability of data and materials The dataset supporting the conclusions of this article is available on request. The authors had full access to all of the data and had the right to publish it on the basis of their own decision only.

Competing interests The authors declare that they have no competing interests.

Funding This research received no specific grant.

Open Access This article is distributed under the terms of the Creative Commons Attribution 4.0 International License (http:// creativecommons.org/licenses/by/4.0/), which permits unrestricted use, distribution, and reproduction in any medium, provided you give appropriate credit to the original author(s) and the source, provide a link to the Creative Commons license, and indicate if changes were made.

\section{References}

1. Natoli JL, Manack A, Dean B, Butler Q, Turkel CC, Stovner L, Lipton RB (2010) Global prevalence of chronic migraine: a systematic review. Cephalalgia 30:599-609

2. Olesen J (2013) Headache Classification Committee of the International Headache Society (IHS): The International Classification of Headache Disorders, $3^{\text {rd }}$ edition (beta version). Cephalalgia 33:629-808

3. Bigal ME, Serrano D, Reed M et al (2008) Chronic migraine in the population: burden, diagnosis, and satisfaction with treatment. Neurology 71:559-566

4. Dodick DW (2006) Clinical practice. Chronic daily headache. N Engl J Med 354:158-165

5. Meletiche DM, Lofland JH, Young WB (2001) Quality-of-life differences between patients with episodic and transformed migraine. Headache 41:573-578

6. Duru G, Auray JP, Gaudin AF et al (2004) Impact of headache on quality of life in a general population survey in France (GRIM2000 Study). Headache 44:571-580

7. Osterhaus JT, Townsend RJ, Gandek B, Ware JE Jr (1994) Measuring the functional status and well-being of patients with migraine headache. Headache 34:337-343

8. Rojo E, Pedraza MI, Muñoz I, Mulero P, Ruiz M, de la Cruz C, Barón J, Rodríguez C, Herrero S, Guerrero AL (2015) Chronic migraine with and without medication overuse: experience in a hospital series of 434 patients. Neurología 30:153-157

9. Deiner HC, Limmroth V (2004) Medication overuse headache: a worldwide problem. Lancet Neurol 3:475-483

10. Negro A, D'Alonzo L, Martelletti P (2010) Chronic migraine: comorbidities, risk factors, and rehabilitation. Intern Emerg Med 5(Suppl 1):S13-S19

11. Caproni S, Bianchi E, Cupini ML, Corbelli I, Beghi E, Calabresi P, Sarchielli P, SAMOHA Study Group (2015) Migraine-specific quality of life questionnaire and relapse of medication overuse headache. BMC Neurol 15:85

12. Sarchielli P, Corbelli I, Messina P, Cupini LM, Bernardi G, Bono G, Di Piero V, Petolicchio B, Livrea P, Prudenzano MP, Pini LA, Sandrini G, Allena M, Tedeschi G, Russo A, Caproni S, Beghi E, Calabresi P, SAMOHA Study Group (2016) Psychopathological comorbidities in medication-overuse headache: a multicentre clinical study. Eur J Neurol 23(1):85-91

13. Inc A (2013) BOTOX (onabotulinumtoxinA) full prescribing information. Allergan Inc, Irvine

14. BOTOX_(onabotulinumtoxinA) (2014) full prescribing information. Allergan Inc., Irvine

15. Martelletti $P$, Katsarava Z, Lampl C, Magis D, Bendtsen L, Negro A, Russell MB, Mitsikostas DD, Jensen RH (2014) Refractory chronic migraine: a consensus statement on clinical definition from the European Headache Federation. J Headache Pain 15-47

16. Blumenfeld A, Silberstein SD, Dodick DW, Aurora SK, Turkel CC, Binder WJ (2010) Method of injection of onabotulinumtoxinA for chronic migraine: a safe, well-tolerated, and effective treatment paradigm based on the PREEMPT clinical program. Headache 50: 1406-1418

17. Aurora SK, Dodick DW, Turkel CC, RE DG, Silberstein SD, Lipton RB, Diener HC, Brin MF, PREEMPT 1 Chronic Migraine Study Group (2010) OnabotulinumtoxinA for treatment of chronic migraine: results from the double-blind, randomized placebocontrolled phase of the PREEMPT 1 trial. Cephalalgia 30:793-803

18. Diener HC, Dodick DW, Aurora SK, Turkel CC, DeGryse RE, Lipton RB, Silberstein SD, Brin MF (2010) PREEMPT 2 Chronic Migraine Study Group OnabotulinumtoxinA for treatment of chronic migraine: results from the double-blind, randomized, placebo-controlled phase of the PREEMPT 2 trial. Cephalalgia 30:804-814

19. Dodick DW, Turkel CC, RE DG, Aurora SK, Silberstein SD, Lipton RB, Diener HC, Brin MF, PREEMPT Chronic Migraine Study Group (2010) OnabotulinumtoxinA for treatment of chronic migraine: pooled results from the double-blind, randomized placebo-controlled phases of the PREEMPT clinical program. Headache 50:921-936

20. Negro A, Curto M, Lionetto L, Crialesi D, Martelletti P (2015) OnabotulinumtoxinA $155 \mathrm{U}$ in medication overuse headache: a two years prospective study. Spring 4:826

21. Negro A, Curto M, Lionetto L, Crialesi D, Martelletti P (2016) A two years open-label prospective study of OnabotulinumtoxinA $195 \mathrm{U}$ in medication overuse headache: a real-world experience. J Headache Pain 17:1

22. Guerzoni S, Pellesi L, Baraldi C, Pini LA (2015) Increased efficacy of regularly repeated cycles with OnabotulinumtoxinA in $\mathrm{MOH}$ patients beyond the first year of treatment. J Headache Pain 17:48

23. Cernuda-Morollón E, Ramón C, Larrosa D, Alvarez R, Riesco N, Pascual J (2015) Long-term experience with onabotulinumtoxinA in the treatment of chronic migraine: what happens after one year? Cephalalgia 35:864-868

24. IHS (2013) International classification on headache disorders (ICHD-3 beta). Cephalalgia 33(9):629-808

25. Stewart WF, Lipton RB, Kolodner K, Liberman J, Sawyer J (1999) Reliability of the migraine disability assessment score in a population-based sample of headache sufferers. Cephalalgia 19: 107-113

26. Tana C, Tafuri E, Tana M, Martelletti P, Negro A, Affaitati G, Fabrizio A, Costantini R, Mezzetti A, Giamberardino MA (2013) New insights into the cardiovascular risk of migraine and the role of white matter hyperintensities: is gold all that glitters? J Headache Pain 14:9

27. Bellei E, Monari E, Bergamini S, Bergamini S, Cuoghi A, Tomasi A, Guerzoni S, Ciccarese M, Pini LA (2015) Validation of potential candidate biomarkers of drug-induced nephrotoxicity and allodynia in medication overuse headache. J Headache Pain 16:559

28. Lionetto L, Negro A, Palmisani S, Gentile G, Del Fiore MR, Mercieri M, Simmaco M, Smith T, Al-Kaisy A, Arcioni R, Martelletti P (2012) Emerging treatment for chronic migraine and refractory chronic migraine. Expert Opin Emerg Drugs 17:393-406 
29. Aurora SK, Dodick DW, Diener HC, Aurora SK, Dodick DW, Diener HC, DeGryse RE, Turkel CC, Lipton RB, Silberstein SD et al (2014) Onabotulinumtoxin A for chronic migraine: efficacy, safety and tolerability in patients who received all five treatment cycles in the PREEMPT clinical program. Acta Neurol Scand 129: $61-70$

30. Silberstein SD, Dodick WD, Aurora SK, Dienere H-C, DeGryse RE, Lipton RB, Turkel CC (2015) Per cent of patients with chronic migraine who responded per onabotulinumtoxinA treatment cycle: PREEMPT. J Neurol Neurosurg Psychiatry 86:996-1001

31. Grazzi L, Usai S (2015) Onabotulinum toxin A (Botox) for chronic migraine treatment: an Italian experience. Neurol Sci 36(Suppl 1): S33-S35

32. Pedraza MI, de la Cruz C, Ruiz M, López-Mesonero L, Martínez E, de Lera M, Guerrero ÁL (2015) OnabotulinumtoxinA treatment for chronic migraine: experience in 52 patients treated with the PREEMPT paradigm. Spring 13(4): 176

33. Russo M, Manzoni GC, Taga A, Genovese A, Veronesi L, Pasquarella C, Sansebastiano GE, Torelli P (2016) The use of onabotulinum toxin A (Botox ${ }^{\circledR}$ ) in the treatment of chronic migraine at the Parma Headache Centre: a prospective observational study. Neurol Sci 37:1127-1131
34. De Tommaso M, Franco G, Ricci K, Montemurno A, Sciruicchio V (2016) Laser evoked potentials in early and presymptomatic Huntington's disease. Behav Neurol 1-8. $8 \mathrm{p}$

35. Perrotta A, Serrao M, Sandrini G, Burstein R, Sances G, Rossi P, Bartolo M, Pierrelli F, Nappi G (2010) Sensitisation of spinal cord pain processing in medication overuse headache involves supraspinal pain control. Cephalalgia 30(3):272-284

36. Aoki KR (2005) Review of a proposed mechanism for the antinoceceptive action of botulinum toxin type A. Neurotoxicology 26:785-793

37. Sandrini G, Perrotta A, Tassorelli C, Torelli P, Brighina F, Sances G, Nappi G (2011) Botulinum toxin type-A in the prophylactic treatment of medication overuse headache: a multicentre, double-blind, randomised, placebo-controlled, parallel group study. J Headache Pain 12:427-433

38. Stewart WF, Lipton RB, Kolodner K, Sawyer J, Lee C, Liberman JN (2000) Validity of the Migraine Disability Assessment (MIDAS) score in comparison to a diary-based measure in a population sample of migraine sufferers. Pain 88:41-52

39. Stewart AL, Lipton RB, Whyte J et al (1999) A multinational study to assess reliability of the Migraine Disability Assessment (MIDAS) score. Neurology 53:988-994 